\title{
Singularity-free electrodynamics for point charges and dipoles: a classical model for electron self-energy and spin
}

\author{
S M Blinder \\ University of Michigan, Ann Arbor, MI 48109-1055, USA \\ E-mail: sblinder@umich.edu
}

Received 9 December 2002, in final form 17 February 2003

Published 10 March 2003

Online at stacks.iop.org/EJP/24/271

\begin{abstract}
It is shown how point charges and point dipoles with finite self-energies can be accommodated in classical electrodynamics. The key idea is the introduction of constitutive relations for the electromagnetic vacuum, which actually mirrors the physical reality of vacuum polarization. Our results reduce to conventional electrodynamics for scales large compared to the classical electron radius $r_{0} \approx 2.8 \times 10^{-15} \mathrm{~m}$. A classical simulation for a structureless electron is proposed, with the appropriate values of mass, spin and magnetic moment.
\end{abstract}

\section{Introduction}

The most elementary problems in classical electrodynamics are likely to involve point charges, point dipoles and other tractable mathematical idealizations of physical reality. But the simplicity of these solutions often obscures a subtle but pervasive conceptual flaw. This appears even in the most elementary formula of electrostatics, Coulomb's law of force between two point charges $q_{1}$ and $q_{2}$ separated by a distance $r$ [1]

$$
F=\frac{q_{1} q_{2}}{4 \pi \epsilon_{0} r^{2}}
$$

with the corresponding energy of interaction

$$
W=\frac{q_{1} q_{2}}{4 \pi \epsilon_{0} r} .
$$

What if we want to know the hypothetical force a point charge would exert on itself? Since we would then have $r=0$ in these equations, this force becomes infinitely large, as does the self-energy - the energy of interaction of the point charge with its own electric field. For the most part, these difficulties have been 'swept under the rug' since electrically charged bodies in real life have a finite size. A point charge is perhaps just an abstraction. Still, it would be highly desirable, even for purely aesthetic reasons, to remove this imperfection from the otherwise 
beautiful and complete edifice of Maxwell's electrodynamics. Dimensionless point charges are in fact the paradigm for representing fundamental particles in quantum mechanics and quantum field theory. And all experimental evidence from high-energy physics appears to support such models. For example, recent high-energy electron-positron scattering experiments imply an upper limit of $2 \times 10^{-18} \mathrm{~m}$ on the radius of the electron.

The strategy we pursue invokes constitutive relations. Usually, these are phenomenological parameters which represent properties of matter, serving as inputs to Maxwell's equations, not implied by the stucture of electrodynamics itself. In certain favourable instances, these parameters can be determined theoretically from quantum theories of matter. The idea which we will exploit is to attribute constitutive properties to the vacuum. According to quantum field theory, the ultramicroscopic vicinity of an elementary charged particle is a seething maelstrom of virtual electron-positron pairs (and other particles and antiparticles) flashing in and out of existence. To take account of this well established physical reality, a phenomenological representation for vacuum polarization is introduced within the framework of classical electrodynamics. As we will show, such a model enables a consistent picture of classical point charges with finite electromagnetic self-energy. We must emphasize that the model is intended in a purely classical context and will not necessarily be in agreement with details of quantum electrodynamics. In the same sense, continuum models for dielectric media can be extremely successful without taking account of the underlying atomic nature of matter.

\section{Self-energy of a point charge}

The energy contained in an electromagnetic field is given by

$$
W=\frac{1}{2} \int(\boldsymbol{E} \cdot \boldsymbol{D}+\boldsymbol{B} \cdot \boldsymbol{H}) \mathrm{d}^{3} \boldsymbol{r} .
$$

In a rest frame, the field produced by a point charge $e$ in vacuum is represented by $\boldsymbol{D}=e \hat{\boldsymbol{r}} / 4 \pi r^{2}, \boldsymbol{E}=e \hat{\boldsymbol{r}} / 4 \pi \epsilon_{0} r^{2}, \boldsymbol{B}=\boldsymbol{H}=0$. For concreteness, let the particle be an electron. The electromagnetic self-energy is then given by

$$
W=\frac{1}{32 \pi^{2} \epsilon_{0}} \int \frac{e^{2}}{r^{4}} 4 \pi r^{2} \mathrm{~d} r=\infty
$$

The result is infinite unless a lower cut-off is introduced-in which case the electron acquires a finite size, as in the models proposed by Thomson, Lorentz, Abraham and others a century ago [2]. With a radius of the order of $r_{0}=e^{2} / 4 \pi \epsilon_{0} m c^{2} \approx 2.818 \times 10^{-15} \mathrm{~m}$, known as the classical or Thomson radius, the electromagnetic self-energy can be adjusted to equal $m c^{2}$. This is in accord with the original idea of Lorentz and Abraham that the electron's rest mass is purely electromagnetic in origin. Because of mutual repulsions among the electron's elements of charge, the whole structure might be expected to blow itself apart. Poincaré postulated the existence of nonelectromagnetic attractive forces - later called Poincaré stresses - to somehow counterbalance the Coulomb repulsion.

It was suggested in the 1930s by Furry and Oppenheimer [3] that quantum-electrodynamic effects could give the vacuum some characteristics of a polarizable medium, which Weisskopf [4] represented phenomenologically by an inhomogeneous electric permittivity, namely,

$$
\boldsymbol{D}(r)=\epsilon(r) \boldsymbol{E}(r) \text {. }
$$

Thus, assuming the electron's rest mass is entirely electromagnetic,

$$
W=\frac{1}{32 \pi^{2}} \int_{0}^{\infty} \frac{1}{\epsilon(r)} \frac{e^{2}}{r^{4}} 4 \pi r^{2} \mathrm{~d} r=m c^{2} .
$$

The net charge density $\rho(r)$, taking account of the conjectured vacuum polarization, is given by

$$
\nabla \cdot \boldsymbol{E}=-\frac{e \epsilon^{\prime}(r)}{4 \pi r^{2}[\epsilon(r)]^{2}}=\frac{\rho(r)}{\epsilon_{0}} .
$$


The original point charge is here exactly cancelled by a delta-function contribution from the polarization charge. A functional form for $\epsilon(r)$ can be determined if the charge density $\rho(r)$ is assumed to be proportional to the electromagnetic energy density, so that

$$
-\frac{\epsilon_{0} \epsilon^{\prime}(r)}{4 \pi r^{2}[\epsilon(r)]^{2}}=\frac{e^{2}}{32 \pi^{2} m c^{2} \epsilon(r) r^{4}} .
$$

The result is [5]

$$
\epsilon(r)=\epsilon_{0} \exp \left(\frac{e^{2}}{8 \pi \epsilon_{0} m c^{2} r}\right)=\epsilon_{0} \exp \left(\frac{r_{0}}{2 r}\right) .
$$

The self-energy then follows from

$$
W=\frac{e^{2}}{8 \pi \epsilon_{0}} \int_{0}^{\infty} \frac{\mathrm{e}^{-r_{0} / 2 r}}{r^{2}} \mathrm{~d} r=\frac{e^{2}}{4 \pi \epsilon_{0} r_{0}}=m c^{2} .
$$

\section{Self-energy of a dipole}

A point electric dipole $\mathfrak{p}$ located at the origin and directed along the polar axis produces an axially symmetric field given by

$$
\boldsymbol{D}=\frac{2 \mathfrak{p} \cos \theta}{4 \pi r^{3}} \hat{\boldsymbol{r}}+\frac{\mathfrak{p} \sin \theta}{4 \pi r^{3}} \hat{\boldsymbol{\theta}} .
$$

If it is assumed that the permittivity $\epsilon(r)$ remains spherically symmetrical, the field energy integrated over the solid angle is given by

$$
\frac{1}{2} \int_{0}^{2 \pi} \int_{0}^{\pi} \boldsymbol{E} \cdot \boldsymbol{D} \sin \theta \mathrm{d} \theta \mathrm{d} \phi=\frac{\mathfrak{p}^{2}}{4 \pi r^{6} \epsilon(r)} .
$$

The charge density analogous to $(7)$ is determined by the proportionality

$$
\rho(r)=\epsilon_{0} \nabla \cdot \boldsymbol{E} \approx-\frac{\epsilon^{\prime}(r)}{r^{3}[\epsilon(r)]^{2}}
$$

so that the relation analogous to (8) implies a permittivity of the form

$$
\epsilon_{\text {dipole }}(r)=\epsilon_{0} \exp \left(k^{2} / r^{2}\right)
$$

where $k$ is a parameter with dimensions of length. For example, a hypothetical electric dipole $\mathfrak{p}$ with electromagnetic self-energy $M c^{2}$ would imply

$$
k=\left(\frac{\mathfrak{p}^{2}}{16 \sqrt{\pi} \epsilon_{0} M c^{2}}\right)^{1 / 3} .
$$

The treatment of a magnetic dipole $\mathfrak{m}$ is closely analogous. The magnetic field in vacuum is given by

$$
\boldsymbol{H}=\frac{2 \mathfrak{m} \cos \theta}{4 \pi r^{3}} \hat{\boldsymbol{r}}+\frac{\mathfrak{m} \sin \theta}{4 \pi r^{3}} \hat{\boldsymbol{\theta}} .
$$

Assuming a spherically symmetrical magnetic permeability, we have

$$
\boldsymbol{B}=\mu(r) \boldsymbol{H} .
$$

In analogy with (12), the magnetic field energy integrated over the solid angle is given by

$$
\frac{1}{2} \int_{0}^{2 \pi} \int_{0}^{\pi} \boldsymbol{B} \cdot \boldsymbol{H} \sin \theta \mathrm{d} \theta \mathrm{d} \phi=\frac{\mathfrak{m}^{2} \mu(r)}{4 \pi r^{6}} .
$$

The current density of polarized charge can be found from

$$
\boldsymbol{J}=\frac{1}{\mu_{0}} \nabla \times \boldsymbol{B} \approx \frac{\mu^{\prime}(r)}{r^{3}} .
$$


An analogous assumption that the magnetic energy density is proportional to the polarization current density thus implies a magnetic susceptibility of the form

$$
\mu(r)=\mu_{0} \exp \left(-b^{2} / r^{2}\right) .
$$

Since $\epsilon(r) \rightarrow \infty$ and $\mu(r) \rightarrow 0$ as $r \rightarrow 0$, both $\boldsymbol{E} \cdot \boldsymbol{D}$ and $\boldsymbol{B} \cdot \boldsymbol{H}$ vanish at the origin. Thus we need not consider contact contributions of the form $\mathfrak{p} \delta(\boldsymbol{r})$ or $\mathfrak{m} \delta(\boldsymbol{r})$.

\section{Classical model for the electron}

Let the electron be pictured as a structureless point charge $e$ with a magnetic dipole moment $\mathfrak{m}=e \hbar / 2 m$. If the energy is entirely electromagnetic, according to Lorentz and Abraham, the intrinsic angular momentum should likewise be electromagnetic. This suggests a possible rationalization for the existence of spin angular momentum in a dimensionless particle. (Alternatively, this might be attributed to the motion of the polarization charge surrounding the electron.) The angular momentum of an electromagnetic field is given by

$$
\boldsymbol{S}=\frac{1}{c^{2}} \int \boldsymbol{r} \times(\boldsymbol{E} \times \boldsymbol{H}) \mathrm{d}^{3} \boldsymbol{r} .
$$

Identifying this with the electron's spin of one-half, we can write

$$
S_{z}=\frac{1}{c^{2}} \int r \sin \theta(\boldsymbol{E} \times \boldsymbol{H})_{\phi} \mathrm{d}^{3} \boldsymbol{r}=\frac{\hbar}{2} .
$$

Using the fields

$$
\boldsymbol{D}=\frac{e}{4 \pi r^{2}} \hat{\boldsymbol{r}}, \quad \boldsymbol{E}=\frac{\boldsymbol{D}}{\epsilon(r)}, \quad \boldsymbol{H}=\frac{2 \mathfrak{m} \cos \theta}{4 \pi r^{3}} \hat{\boldsymbol{r}}+\frac{\mathfrak{m} \sin \theta}{4 \pi r^{3}} \hat{\boldsymbol{\theta}}
$$

with

$$
\mathfrak{m}=\frac{e \hbar}{2 m}
$$

and the permittivity parametrized as

$$
\epsilon(r)=\epsilon_{0} \mathrm{e}^{a / r},
$$

equation (21) is satisfied with

$$
a=\frac{e^{2}}{6 \pi \epsilon_{0} m c^{2}}=\frac{2}{3} r_{0} .
$$

The electric-field energy then works out to

$$
W_{\text {elec }}=\frac{1}{2} \int \boldsymbol{E} \cdot \boldsymbol{D} \mathrm{d}^{3} \boldsymbol{r}=\frac{3}{4} m c^{2} .
$$

The magnetic contribution must then supply the remaining quarter of the rest energy:

$$
W_{\text {mag }}=\frac{1}{2} \int \boldsymbol{B} \cdot \boldsymbol{H} \mathrm{d}^{3} \boldsymbol{r}=\frac{1}{4} m c^{2} .
$$

With the parametrization $\mu(r)=\mu_{0} \exp \left(-b^{2} / r^{2}\right)$, equation (27) is satisfied with

$$
b=\left(\frac{\mathfrak{m}^{2} \mu_{0}}{4 \sqrt{\pi} m c^{2}}\right)^{1 / 3}=\frac{\pi^{1 / 6}}{2^{2 / 3} \alpha^{2 / 3}} r_{0}
$$

where $\alpha=e^{2} / 4 \pi \epsilon_{0} \hbar c$, the fine structure constant. 


\section{Conclusion}

We have shown how to accommodate point structures with finite self-energies in classical electrodynamics without altering the equations of Maxwell's theory. This is in contrast to the earlier attempts of Born [6], Bopp [7] and others, which involved nonlinear reformulations of the fundamental equations. The key to our approach is the introduction of constitutive parameters for the electromagnetic vacuum, which actually has a physical rationale according to quantum field theory. In any event, our results reduce smoothly to conventional electrodynamics for scales large compared to $10^{-15} \mathrm{~m}$. In particular, $\epsilon(r)$ and $\mu(r)$ both rapidly approach their vacuum values of $\epsilon_{0}$ and $\mu_{0}$, respectively.

The Lorentz-Abraham conjecture, that the electron's rest mass of $0.511 \mathrm{MeV} / c^{2}$ is entirely electromagnetic, is made more plausible by the model we have described. This is consistent as well with the (nearly, if not exactly) zero rest mass of the electron's uncharged weak isodoublet partner-the neutrino-which can be regarded as an electron with zero charge. We also note that the neutron-proton mass difference $\left(1.29 \mathrm{MeV} / c^{2}\right)$ is of comparable order of magnitude. The parameters which we have fitted to the electron's mass, angular momentum and magnetic moment imply a $g$-factor of 2, consistent with Dirac's relativistic theory. (We have resisted the temptation to adjust this to 2.0023, with QED radiative corrections.)

Of course, the real physical electron must ultimately be described by quantum mechanics or quantum field theory. Still, a fully consistent classical model can provide a useful starting point [8], and classical results do (usually) represent $\hbar \rightarrow 0$ limits in quantum theory. Since it is by no means settled that the current formalism of quantum electrodynamics is the final theory of the electron, it is worthwhile to explore the classical limit that some successor theory might also exhibit. Although the infinities associated with transverse radiation fields do remain, we have succeeded in eliminating those of classical origin for a point charge.

\section{References}

[1] SI units are used in this paper. For results expressed in Gaussian units see http://arxiv.org/abs/physics/0208072

[2] A definitive review of classical electron theories is given by Rohrlich F 1990 Classical Charged Particles (Reading, MA: Addison-Wesley)

[3] Furry W and Oppenheimer J R 1934 Phys. Rev. 45245

[4] Weisskopf V F 1936 Det. Kgl. Danske Videnskab. Selskab. Mat.-Fys. Medd. 141 Schwinger J 1958 Quantum Electrodynamics (New York: Dover) (Reprint)

[5] Blinder S M 2001 Rep. Math. Phys. 47269 Online version: http://arXiv.org/find/physics/1/au:+Blinder/0/1/0/past/0/1

[6] Born M 1934 Proc. R. Soc. A 143410 Born M and Infeld L 1934 Proc. R. Soc. A 144425

[7] Bopp F 1940 Ann. Phys., NY 38345

[8] The connection between classical and quantum theories of the electron is discussed by Pearle P 1982 Classical electron models Electromagnetism: Paths to Research ed D Teplitz (New York: Plenum) pp 211-95 